\title{
Sports Training Decision Making Method Based on AHP and Evidence Theory \\ Bi Yao ${ }^{1}$, Wang Qingbin ${ }^{1}$ \\ 1.Zhuhai College of Jilin University, Zhuhai, 519000, china
}

Keywords: Sports; decision support; training program; evidence theory

\begin{abstract}
As one of the highlights in Olympics, sports events always have significant impact on Olympic Strategies due to their levels and have become key programs in the world. Sports events in China have made further development in recent years and created outstanding achievements in many national and international competitions, however, traditional decision making based on experience during training still dominated, while more qualitative and less quantitative decision methods have limited the development of sports in China. The sports fencing combining with modern and advanced computer technology and intelligent, scientific and quantitative training has become not only the development trend of world sports, but also the goal of Chinese sports trainers. Thus, it is necessary to establish a supporting system of sports training decisions making, which could meet normal and simple inquiry and maintenance needs, also able to provide deep information of competitive ability, training direction and historical data of athletes to sports team coaches and leaders for their decision making. Therefore, it is particularly important to figure out how to create a data warehouse, how to take use of existing data and how to make higher level of processing - data mining (DM) on that basis.
\end{abstract}

\section{Introduction}

Since traditional sports training management methods and plans were determined by man on their own, errors are unavoidable and rationality and scientificity of such methods and plans have been increasingly questioned. Those plans and methods failed to engage with physical fitness of students and were unable to achieve desirable training effect; students had no enthusiasm in sports training, so their physical fitness was limited. That is why this topic arose. One of topic objective is to create a sport training decision making method based on AHP and evidence theory. It will improve the problems which college students faced in sports training management and determine the scientific and reasonable training methods and plans that are truly suitable for physical quality of college students, which could not only avoid unnecessary mistakes and impairment caused by human factors during traditional training management, but also make the sports training process more impairment and efficacious.

\section{Grey correlation method on entropy weight}

Information entropy weighting method takes entropy definition in information theory as basis and creates an indicator weight determining method by using of indicator value, which is relatively objective and avoids subjectivity and blindness of weight set by man. Grey correlation method has certain advantages when information processing was incomplete, biased and inadequate.

Determination of indicator weight

In a complicated environment, there are many factors could affect sports and the following selected training plans indicators are major objective of decision making. According to each indicator data information of opponents during training and combining with related parameters of training goal data in data warehouse of our athletes, the degree of membership of decision i under indicator $\mathrm{j}$ could be measured, thus to form a goal membership degree matrix $G=\left(g_{i j}\right)_{m \times n}$.

Use entropy theory to determine each indicator weight, thus to determine an entropy method for evaluation object which has $\mathrm{i}$ objects and $\mathrm{j}$ indicators, which are as follows: 
Normalize $G=\left(g_{i j}\right)_{m \times n}$ and we can get:

Entropy value of indicator $\mathrm{j}$ is:

$$
Y=\left(y_{i j}\right)_{m \times n}=\left(g_{i j} / \sum_{i=1}^{m} g_{i j}\right)_{m \times n}
$$

$$
E_{j}=-1 / \ln n \sum_{i=1}^{m} y_{i j} \ln y_{i j}
$$

Weight of indicator $\mathrm{j}$ is:

$$
\omega_{j}=\left(1-E_{j}\right) /\left(n-\sum_{j=1}^{n} E_{j}\right)
$$

In which, $0 \leq \omega_{j} \leq 1$ and $\sum_{j=1}^{n} \omega_{j}=1$, substitute $\left(y_{i j}\right)_{m \times n}$ with $\omega_{j}(j=1,2, \ldots n)$ and we can get the weight membership degree matrix as follows:

$$
X=\left(x_{i j}\right)_{m \times n}=\left(\omega_{j} \cdot y_{i j}\right)_{m \times n}
$$

Grey correlation method to determine Mass function

When using D-S evidence theory to make decision, information of different objects under each indicator shall be integrated, and Mass function is the basis for that. Solution of uncertainty of each indicator in an important step for Mass function and we will apply grey correlation method to obtain the uncertainty of each indicator below.

We assume that $q$ order uncertainty under indicator $\mathrm{j}$ is:

$$
\operatorname{DOI}\left(I_{j}\right)=\frac{1}{m}\left|\sum_{i=1}^{m}\left(r_{i j}\right)^{q}\right|^{\frac{1}{q}}
$$

In which, assume that $\mathrm{q}=2$ and $r_{i j}$ is the Integrated Grey Correlation Coefficient of $\left(x_{i j}\right)_{m \times n}$. The application of Integrated Correlation method to obtain Correlation Coefficient could avoid the distorted effects caused by individual application of optimal or worst correlation and promote accuracy of Grey Correlation. Solution of ${ }^{r_{i j}}$ is as follows, firstly, we assume the optimal coefficient $r_{i j}^{+}$and worst correlation coefficient ${ }^{r_{i j}^{-}}$as:

$$
\begin{aligned}
r_{i j}^{+} & =\frac{\min _{i} \min _{j}\left|x_{i j}-X^{+}\right|+\xi \max _{i} \max _{j}\left|x_{i j}-X^{+}\right|}{\left|x_{i j}-X^{+}\right|+\xi \max _{i} \max _{j}\left|x_{i j}-X^{+}\right|} \\
r_{i j}^{-} & =\frac{\min _{i} \min _{j}\left|x_{i j}-X^{-}\right|+\xi \max _{i} \max _{j}\left|x_{i j}-X^{-}\right|}{\left|x_{i j}-X^{-}\right|+\xi \max _{i} \max _{j}\left|x_{i j}-X^{-}\right|}
\end{aligned}
$$

In which is

$$
X^{+}=\max _{\substack{1 \leq i \leq m \\ 1 \leq j \leq n}} x_{i j}=\left\{x_{1}^{+}, \ldots, X_{n}^{+}\right\}
$$

is the ideal optimal sequence and $X^{-}=\min _{\substack{1 \leq i \leq m \\ 1 \leq j \leq n}} x_{i j}=\left\{x_{1}^{-}, \ldots, x_{n}^{-}\right\}$ is the ideal worst sequence, assume $\xi=0.5$ and the Integrated Grey Correlation Coefficient can be expressed as:

$$
r_{i j}=\frac{1}{\left(1+r_{i j}^{+} / r_{i j}^{-}\right)^{2}}
$$

Bring ${ }^{r_{i j}}$ into formula (5) to obtain uncertainty $D O I\left(I_{j}\right)$ under each indicator. Through formula (9) we can determine the Mass function of different objects under each indicator:

$$
m_{j}(i)=\left[1-\operatorname{DOI}\left(I_{j}\right)\right] y_{i j}
$$

In which, $m_{j}(i)$ is the Mass function of indicator $\mathrm{j}$ under object $\mathrm{i}$ and $\sum_{i=1}^{m} m_{j}(i)<1$, means uncertainty on overall understanding exists, assign this part of Mass function to identification framework $\Theta$ means its supporting degree to all objects and can participate in integrated calculation for purpose to reduce the uncertainty during decision making. Thus, we can obtain the overall uncertainty Mass function under indicator $\mathrm{j}$ as follows: 


$$
m_{j}(i+1)=1-\sum_{i=1}^{m} m_{j}(i)
$$

\section{System design}

Sports training are known as exploratory practice that taking winning as final goal and running with multi-disciplinary intervention and multi-system coordination. The fundamental structure model of it can be expressed as shown in Figure 1. In which coaches will let the decision supporting system to process the external information (latest trends at home and abroad, information of excellent athletes) and basic information of athlete (initial state diagnosis, current quality, skills, and development potential in sports) and determine training plans on that basis, send such plans to athletes in manner of command information, athletes will conduct specific practice according to such command information and feedback the practice effect to their coaches, and coaches will adjust the training plans according to the analysis results made by the decision supporting system.

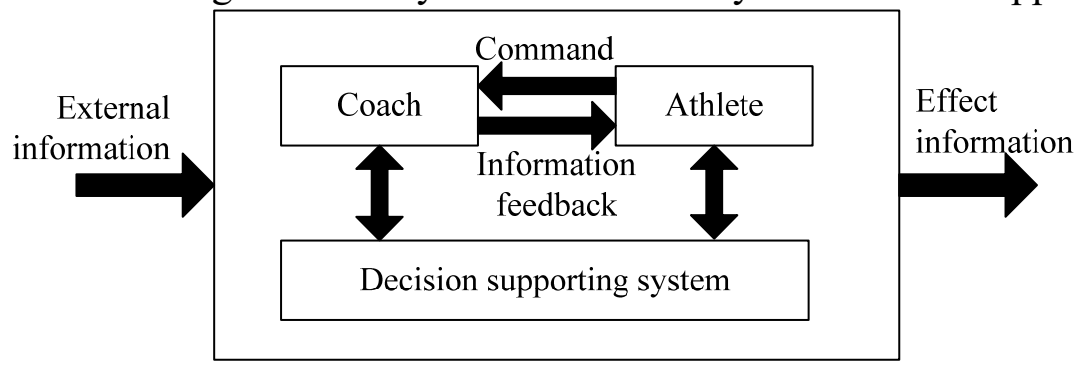

Figure 1 Structural Model of Sports Training Decision Supporting System

The overall program design of sports training decision supporting system structure is guided by the overall design thought of decision supporting system, applies multi-base cooperative technology, which could make knowledge base, model library and database running in integration and achieve diagnosis, evaluation and training through the same interface management. The initial interface of controlling system and each functional system is made up by four subsystem modules, which are Athlete Integrated Information Management System, Athlete Evaluation System, Sports Training Monitoring System and the Management System, in which HMI System is the core and key point for development. HMI System is completed by window mode, pull-down menu mode, table fill method and Q/A method (Question and Answer). User Interface Subsystem is managed by user interface management system (UIMS), which consists of several applications, including the dialogue generation and management system.

Data involved in sports training system is complex and diverse, in order to make it convenient for users to maintain and inquire data and use such data to solve problems, a reasonable planning on database has been completed and in order to obtain coordination of data storage and problem solving, following efforts have been made:

Hierarchical planning of database, in which we divided data into four hierarchies:

(1)Historical state data, reflecting the athletic ability state, historical state of physical quality and the competition records of athletes;

(2)Decision supporting data, means intermediate data and result data during problem solving, such as future development trend of a certain athlete and generated training program;

(3)Decision data: Means the training programs and plans made by the coaches after qualitative and quantitative analysis;

(4)Implement trace data: means the trace feedback information during implementation of training programs, including implementation situation, effect and improvement actions.

Building a model library is essential in sports training system, because the basis of training methods for those super-class athletes is the forecasting method, thus a targeted forecasting model must be created and used: The individual routine model of outstanding athletes, the teaching training model, the training loading model, the physical quality training level and competitive ability model. A time-effect evaluation model corresponding to above models shall also be created: 
physical quality evaluation model of athletes at different levels, competitive ability evaluation model, as well as some other monitoring models that are specialized in sports training. We store those models in model library so as to achieve the decision supporting integration of sports training system. The structure of model library could be described with Data Dictionary as: $W=\{N, F, I$, $\mathrm{A}\}$, in which $\mathrm{W}$ means the name of model library, $\mathrm{N}$ means the model name, $\mathrm{F}$ means the model function and I means the information of model conditions and A means the model algorithm. The overall design of graphics and graphics library as well as the multi-base coordinative and optimal design were also conducted.

The system consists of Athlete Integrated Information Management System, Athlete Evaluation System, Sports Training Monitoring System and the Management System, which are the four main function modules that prop up the decision making of the whole system. They complement each other and achieve their own function by scheduling unified data resources, model resources and methods. Athlete Integrated Information Management System consists of Affair Management and Information Management, which are used to manage large amount of information needed to collect, store and process during sports training. The system is completed with modular design, which makes it easier for completed storage, update, inquiry and report output operations.

\section{Conclusion}

The Essay starts from the present situation of domestic and foreign data mining as well as the sports development, discusses the necessity and inevitability to build the decision supporting system in sports world. On that basis, it proposes the specific way and methods of decision supporting system during sports training, namely conducting data mining on the basis of data warehouse, conducts specific analysis on the package process development methods of specific design and development of such system by using of SQL Server 2000. Practice shows that the application of package process development methods could achieve effectively module reuse, system expansion and maintenance, which might after all be accepted as a better method for system design and development.

\section{Reference}

[1] Yan H E, Xiong C Q. Improvement of the Decision-making Method Based On DS/AHP[J]. Journal of Hubei University of Technology, 2013.

[2] Bachman $\mathrm{J}$ T. Using decision-making techniques in support of simulation training transfer selections[J]. Dissertations \& Theses - Gradworks, 2012.

[3] Onar S C, Oztaysi B, Kahraman C. Strategic Decision Selection Using Hesitant fuzzy TOPSIS and Interval Type-2 Fuzzy AHP: A case study[J]. International Journal of Computational Intelligence Systems, 2014, 7(5):1002-1021.

[4] Tolga Kaya, Cengiz Kahraman. A fuzzy approach to e-banking website quality assessment based on an integrated AHP-ELECTRE method[J]. Technological \& Economic Development of Economy, 2011, 17(2):313-334.

[5] Beynon M, Cosker D, Marshall D. An expert system for multi-criteria decision making using Dempster Shafer theory[J]. Expert Systems with Applications, 2001, 20(4):357-367. 